\title{
Model Predictive Control of Low-Speed Partial Stroke Operated Digital Displacement Pump Unit
}

\author{
N. H. Pedersen \\ P. Johansen \\ A. H. Hansen \\ T. O. Andersen
}

Fluid Power and Mechatronic Systems, Department of Energy Technology, Aalborg University, Pontoppidanstraede 111, 9220 Aalborg, Denmark. E-mail: nhp@et.aau.dk, pjo@et.aau.dk, ahh@et.aau.dk, toa@et.aau.dk

\begin{abstract}
To enhance the use of the Digital Displacement Machine (DDM) technology as the future solution for low speed fluid power pump and motor units, a Model Predictive Control (MPC) strategy is presented. For a low speed DDM, the conventional full stroke operation strategy is unsuitable, since the control update rate is proportional to the machine speed. This creates an incentive to utilize sequential partial stroke operation where a fraction of the full stroke is used, which thereby increases the control update rate and control signal resolution. By doing this, the energy loss is increased and may become undesirable large if the control objective is purely set-point tracking, why a trade-off is considered advantageous. Discretizing the full stroke based on a chosen update rate results in a Discrete Linear Time Invariant (DLTI) model of the system with discrete input levels. In this paper, the Differential Evolution Algorithm (DEA) is used to determine the optimal control input based on the trade-off between set-point tracking and energy cost in the prediction horizon. The paper presents a flow and a pressure control strategy for a fixed speed digital displacement pump unit and shows the trade-off influence on the optimal solution through simulation. Results show the applicability of the control strategy and indicate that a much higher energy efficiency may be obtained with only a minor decrease in tracking performance for pressure control.
\end{abstract}

Keywords: Digital Displacement Units, Fluid Power, Control, Non-smooth System, Hybrid Systems

\section{Introduction}

The Digital Displacement Machine (DDM) technology is a promising alternative to conventional hydraulic pump and motor units, since it provides excellent redundancy and scalability due to its modular construction. Additionally, it provides the possibility of an improved energy efficiency and reliability Linjama (2011). As a result, a large amount of research regarding design and performance optimization of these machines has been published Payne et al. (2005); M. Ehsan and Salter (March 2000); Rampen (2010); Johansen (2014); Roemer (2014); Noergaard (2017); Wilfong et al. (2010, 2011); Merrill et al. (2011).

One important task for successful deployment of the machine technology is the control system, which is a key feature with respect to proper operation and energy efficiency. However, the challenges with respect to control of such non-smooth dynamical system are many and considered to be complicated Pedersen et al. (2018a). Several research papers regarding control strategies of digital displacement machines has been published, but the strategies are often limited to open-loop control at simplified operation conditions M. Ehsan and Salter (March 2000); Heikkila and Linjama (2013); Song (2008); Armstrong and Yuan (June 2006). Control strategies for high speed operated machines have further been exploited to include closed loop control. Sniegucki et al. (2013) presents a mixed logical dynamic programming control structure for a fixed-speed machine. Although the method is limited to offline optimization, promising result is ob- 
tained with respect to fluctuations and set-point tracking. A full stroke Pulse-Density Modulation (PDM) technique is proposed by Johansen et al. (2015) in a feedback structure. To allow for model based closed loop control design, a Discrete Linear Time Invariant (DLTI) approximation to the digital machine dynamics is developed by Johansen et al. (2017) for constant speed operation. To expand the use of this method to be applicable for variable speed operation, Pedersen et al. (2017a) transform the DLTI model to the spatial domain, resulting in a fixed angle sampling rate allowing for classical control synthesis. A combination of the PDM technique and the DLTI model is further used for control of a digital fluid power transmission by Pedersen et al. (2017b, 2018b).

This paper proposes a closed-loop control strategy for a slow rotating digital displacement machine with a relatively low number of cylinders. Because of this, full stroke operation is considered unfavorable, since the control update rate is proportional to the speed of the machine and the tracking performance thus suffers heavily. To account for this, a sequential partial stroke operation method is used with a higher control update rate, which increases the control signal resolution and thereby allows for an improved set-point tracking. Since energy loss due to switching may be of major concern using sequential partial stroke, a Model Predictive Control (MPC) strategy is used. This allows for specifying the importance of tracking performance relative to energy consumption, from where the optimal control input is determined based on a prediction model. The MPC control strategy is tested for both flow and pressure control by simulation in a non-linear model representing the physical system. A fixed speed pump unit is considered in this paper, but the strategy is also applicable to variable speed pump-motor units by the spatial domain transformation method presented by Pedersen et al. (2017a).

\section{System Description}

To evaluate on the performance of the control strategy, a non-linear mathematical model of the digital displacement machine is established. The model hence represents the physical system and is derived based on a description of the machine characteristics. The digital displacement machine under consideration is illustrated in Fig. 1. The illustration to the left shows the digital displacement machine with 10 cylinders being radially distributed around an eccentric shaft. The flow to and from the pressure chambers is regulated by manipulating the state of the fast switching on/off high and low-pressure valves, HPV and LPV respectively illustrated to the right. Maintaining a closed

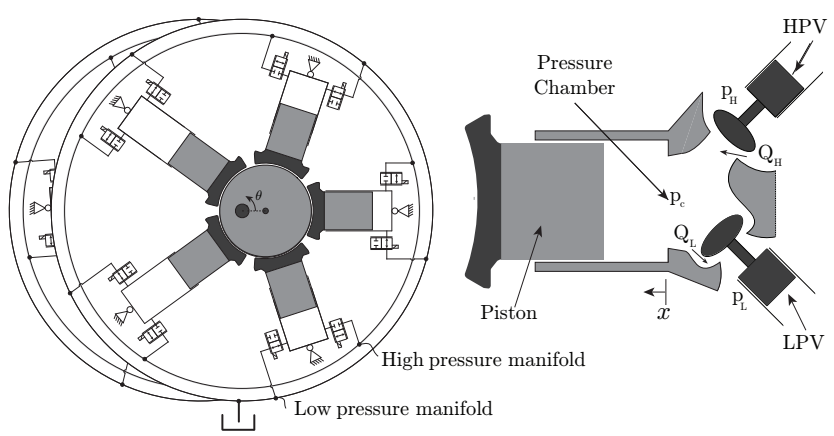

Figure 1: Illustration of the displacement pump-motor unit.

LPV and an open HPV during piston retraction is seen to pressurize the chamber, which result in a flow being pumped to the high pressure manifold. Maintaining the same valve positions results in a motoring mode during piston extension. Using the opposite valve positions results in an idling mode where the chamber pressure remains low. In sequential partial stroke operation, the position of the valves may be changed independently of the cylinder position to obtain a desired volumetric output fraction. However, sequential partial stroke requires that the valves can be opened against high pressure difference. Therefore, larger valves with a higher actuation force are necessary compared to full stroke or conventional partial stroke, where the valve positions may only be altered on a stroke-by-stroke basis.

\subsection{Non-linear Simulation Model}

To evaluate on the control performance, a non-linear simulation model representing the physical system is made. The local shaft angles for the respective cylinders are given by

$$
\theta_{\mathrm{i}}=\theta+\frac{2 \pi}{N_{\mathrm{c}}}(i-1) \quad i \in\left\{1, . ., N_{\mathrm{c}}\right\}
$$

where $\theta$ is the shaft angle and $N_{\mathrm{c}}$ is the number of cylinders. Since the equations used to describe the different pressure chambers are the same, the following model derivation is made for a single chamber. The piston displacement, $x$, is seen to be a function of the shaft angle described as

$$
x(\theta)=r_{\mathrm{s}}(1-\cos (\theta))
$$

where $r_{\mathrm{s}}$ is the eccentric shaft radius being equivalent to half of the piston stroke length. The stroke volume is thus given by $V_{\mathrm{d}}=2 r_{\mathrm{s}} A_{\mathrm{p}}$, where $A_{\mathrm{p}}$ is the piston area. The pressure chamber volume, $V_{\mathrm{c}}$ is then given 
by

$$
\begin{aligned}
& V_{\mathrm{c}}(\theta)=\frac{V_{\mathrm{d}}}{2}(1-\cos (\theta))+V_{0} \\
& \dot{V}_{\mathrm{c}}(\theta)=\frac{V_{\mathrm{d}}}{2} \dot{\theta} \sin (\theta)
\end{aligned}
$$

where $V_{0}$ is the minimum chamber volume. Using the continuity equation to describe the pressure dynamics results in

$$
\dot{p}_{\mathrm{c}}=\frac{\beta_{\mathrm{e}}}{V_{\mathrm{c}}}\left(Q_{\mathrm{H}}-Q_{\mathrm{L}}-\dot{V}_{\mathrm{c}}\right)
$$

The flows through the high and low pressure valve, $Q_{\mathrm{H}}$ and $Q_{\mathrm{L}}$ respectively are modeled by the orifice equation and given by

$$
\begin{aligned}
& Q_{\mathrm{L}}=k_{\mathrm{f}} \sqrt{\left|p_{\mathrm{c}}-p_{\mathrm{L}}\right|} \operatorname{sign}\left(p_{\mathrm{c}}-p_{\mathrm{L}}\right) \bar{x}_{\mathrm{L}} \\
& Q_{\mathrm{H}}=k_{\mathrm{f}} \sqrt{\left|p_{\mathrm{H}}-p_{\mathrm{c}}\right|} \operatorname{sign}\left(p_{\mathrm{H}}-p_{\mathrm{c}}\right) \bar{x}_{\mathrm{H}}
\end{aligned}
$$

where $\bar{x}_{\mathrm{L}}$ and $\bar{x}_{\mathrm{H}}$ are the normalized valve positions of the low- and high-pressure valve respectively and $k_{\mathrm{f}}$ is the valve flow coefficient. To reduce the model complexity significantly, the valve dynamics is simplified to be described as a first order system given by

$$
\dot{\bar{x}}_{\mathrm{L}}=\frac{1}{\tau_{\mathrm{v}}}\left(u_{\mathrm{L}}-x_{\mathrm{L}}\right) \quad \dot{\bar{x}}_{\mathrm{H}}=\frac{1}{\tau_{\mathrm{v}}}\left(u_{\mathrm{H}}-x_{\mathrm{H}}\right)
$$

where $t_{\mathrm{v}}$ is the valve time constant, $u_{\mathrm{L}}$ and $u_{\mathrm{H}}$ are the valve inputs. Despite the simple valve description, the important characteristics with respect to machine level control is captured.

\subsection{Valve timing and control}

The valve actuation is described by considering the hybrid automaton shown in Fig. 2. Two mode switching functions are defined as $\lambda_{\mathrm{H}}=F_{\mathrm{H}}-\left(p_{\mathrm{H}}-p\right) A_{\mathrm{v}}$ and $\lambda_{\mathrm{L}}=F_{\mathrm{L}}-\left(p-p_{\mathrm{L}}\right) A_{\mathrm{v}}$ and are used to construct the switching logic. The systems input is hence the valve forces, $u=\left[\begin{array}{ll}F_{\mathrm{H}} & F_{\mathrm{L}}\end{array}\right]^{T}$ for the high and low pressure valve respectively. $A_{\mathrm{v}}$ is the effective valve area that the pressure is generating a force on. It is seen that the pressure chambers may operate in three modes namely idling, compression and pressurization. The hybrid automaton shows that a pressure chamber is activated if $\lambda_{\mathrm{L}}<-\alpha$, which results in a transition from idling to compression mode. $\alpha$ represents a static force hysteresis band which has to be surmounted in order to alternate the valve state and avoid numerous subsequently switchings. A further transition to the pressurization mode where the HPV is opened is obtained when $\left(p_{\mathrm{H}}-p\right) A_{\mathrm{v}}>\alpha$ or a large force, $F_{\mathrm{H}}$ is applied to the HPV. The valve area $A_{\mathrm{v}}$ is hence considered identically on both sides of the valve plunger as a simplification. Similar transitions occur when deactivating
Deactivate

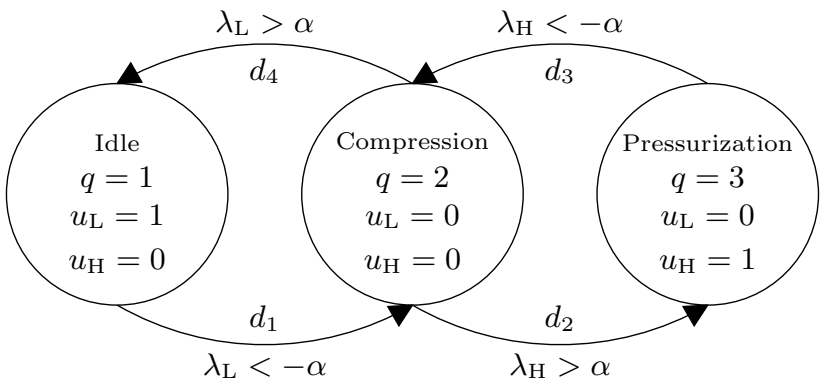

Activate

Figure 2: Hybrid automaton representation of valve control of the digital displacement machine.

a pressure chamber, where the HPV is initially closed and the LPV is subsequently opened.

In conventional partial stroke operation, passive opening of the LPV and HPV due to pressure force is used, such that small valves with a low actuation force may be used. However, the control update is made on a stroke-by-stroke basis and is thus proportional to the machine speed. For a very low speed machine with a low number of cylinders, the control update rate is very low and may be troublesome with respect to obtaining the desired control performance. By using a sequential partial stroke strategy, the valves may be opened and closed independent of the chamber pressure level, but requires valves being able to deliver significantly higher actuation force. Such sequential partial stroke strategy is considered in this paper to significantly increase the control update rate and thereby improve the reference tracking performance. However, due to valve opening against a high pressure difference, the energy loss may also be significantly higher and the strategy is thus only considered feasible for very low speed operation.

The total flow and torque of the digital machine is given as the sum of flows and torques from each pressure chamber and are given by

$$
Q=\sum_{j=0}^{N_{\mathrm{c}}-1} Q_{\mathrm{H}, \mathrm{j}} \quad \tau=\sum_{j=0}^{N_{\mathrm{c}}-1} \tau_{\mathrm{j}}
$$

The parameters used in the simulation model is provided in Tab. 1.

\section{MPC and DLTI model approximation}

Discrete Model Predictive Control (MPC) is characterized as a sample-and-hold feedback control method, where the optimal control input is determined based 
Table 1: Parameters of the 5 MW transmission

\begin{tabular}{l|c|c|c}
\hline Parameter & Symbol & Value & Unit \\
\hline Motor rotation speed & $\omega, \dot{\theta}$ & 10 & $\mathrm{rpm}$ \\
Motor displacement volume & $V_{\mathrm{d}}$ & 153.4 & $\mathrm{~cm}^{3}$ \\
Motor dead volume & $V_{0}$ & 153.4 & $\mathrm{~cm}^{3}$ \\
Maximum bulk-modulus & $\beta_{\mathrm{e}}$ & 16000 & $\mathrm{bar}$ \\
Low pressure & $p_{\mathrm{L}}$ & 10 & $\mathrm{bar}$ \\
Valve flow constant & $k_{\mathrm{f}}$ & 1.26 & $\mathrm{~L} /(\mathrm{min} \sqrt{\mathrm{bar}})$ \\
Valve time constant & $t_{\mathrm{s}}$ & 5 & $\mathrm{~ms}$ \\
Valve effective area & $A_{\mathrm{v}}$ & 31.66 & $\mathrm{~mm}^{2}$ \\
Force threshold & $\alpha$ & 50 & $\mathrm{~N}$ \\
\hline
\end{tabular}

on an estimation of the future plant states in a prediction horizon. Since the system is discretely actuated, a discrete dynamical model is demanded such that the future states may be predicted and the valve switching energy may be included. Two important parameters in MPC is the sampling time and prediction horizon. The sampling rate should be low enough such that the next optimal control input may be calculated between two samples, but high enough to ensure proper control performance. Similar, a small prediction horizon reduces the solving time for the optimal control input but reduces the control performance.

In this paper the optimal control input is chosen as a trade-off between reference tracking accuracy and energy cost. For a digital displacement machine, it is considered advantageously to use a prediction horizon corresponding to the number of samples during one full stroke, $p=5$. This allows the controller to know that it is cheaper to switch the valve states during a low flow at the end of a stroke. Similar, since it is an advantage to switch valve states at the beginning of a stroke at a low flow rate, the sampling time is chosen as the time between two cylinders starting a stroke, $T_{\mathrm{s}}=2 \pi /\left(\omega N_{\mathrm{c}}\right)$. With 10 cylinders this results in 5 samples during a pumping stroke and 5 samples during a motoring stroke, which means that each chamber may switch valve states 5 times during a full stroke.

As long as the valve dynamics is sufficiently fast compared to the machine speed, the switching only takes a neglectable fraction of a full stroke. In this example the valve is switched in $T_{\mathrm{v}} \approx 5 \tau_{\mathrm{v}}=25 \mathrm{~ms}$ and there is $50 \mathrm{~ms}$ from the LPV closing signal is given until the HPV opening signal is given, such that it takes $75 \mathrm{~ms}$ from the LPV closing signal is given until the HPV is fully open. At a speed of $\omega=10 \mathrm{rpm}$ a single revolution takes $6 \mathrm{~s}$, such that it is considered valid to neglect the valve dynamics.

By neglecting the valve and pressure dynamics, a DLTI-model is constructed where $Q_{\mathrm{H}} \approx \dot{V}_{\mathrm{c}}$ during an active stroke. The model is established by considering the change in volume between samples using the relation given by (8) as proposed in Johansen et al.
(2017).

$$
\begin{array}{r}
Q_{\mathrm{d}}[k] \approx \frac{\Delta V_{\mathrm{c}}[k]}{\Delta T[k]} \approx \frac{N_{\mathrm{c}}}{2 \pi} \omega[k] \underbrace{\left(V_{\mathrm{c}}(\theta[k+1])-V_{\mathrm{c}}(\theta[k])\right)}_{\Delta V_{\mathrm{c}}[k]} \\
\tau_{\mathrm{d}}[k] \approx \frac{\Delta V_{\mathrm{c}}[k]}{\Delta \theta} p_{\mathrm{H}}[k] \approx \frac{N_{\mathrm{c}}}{2 \pi} p_{\mathrm{H}}[k] \underbrace{\left(V_{\mathrm{c}}(\theta[k+1])-V_{\mathrm{c}}(\theta[k])\right)}_{\Delta V_{\mathrm{c}}[k]}
\end{array}
$$

where the angle as function of sample number is given by

$$
\theta[k]=\frac{2 \pi}{N_{\mathrm{c}}} k \quad k \in\left\{0, \ldots, N_{\mathrm{c}}-1\right\}
$$

A simulation has been made where the response of the discrete model is compared to that of the non-linear model and is shown in Fig. 3.
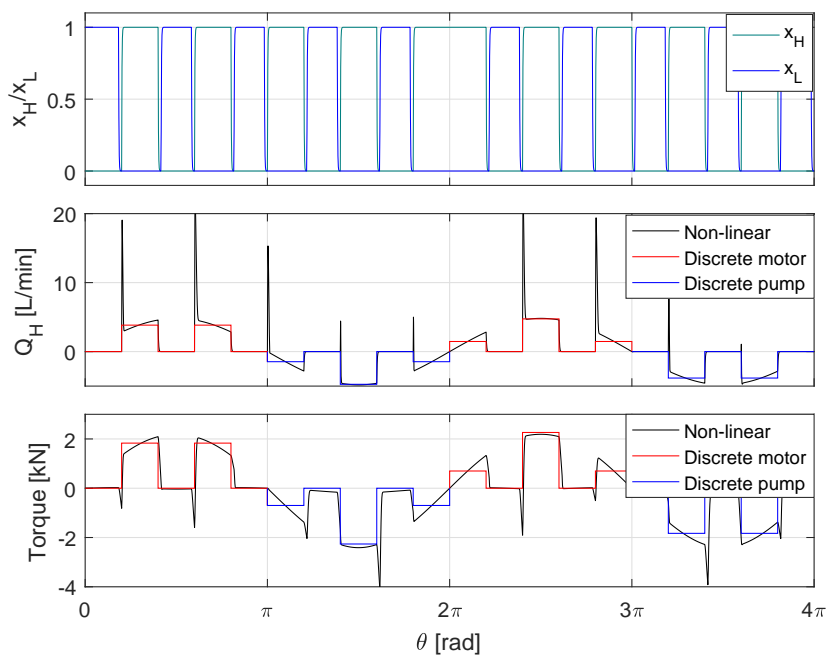

Figure 3: Comparison of non-linear and discrete model of the digital displacement machine.

It is seen that every second decision is to activate the chamber. Although the outputs are approximately matched between samples, large pressure spikes are observed in the non-linear model. These pressure spikes are due to opening against high pressure difference and since the pressure dynamics has been neglected in the discrete model, they are not observed. The severity of the spikes is seen to be larger for higher flow rates and especially high for motoring where the pressure change due to the change in volume is negative. Despite these deviations, the output is matched quite fairly. The total flow and torque throughput of the digital displacement machine is described by a sum of the individual contributions given by

$$
Q[k]=\sum_{m=0}^{N_{\mathrm{c}}-1} Q_{\mathrm{d}}[k] \bar{u}[k] \quad \tau[k]=\sum_{k=0}^{N_{\mathrm{c}}-1} \tau_{\mathrm{d}}[k] \bar{u}[k]
$$

where $\bar{u} \in\{1,0\}$ are the binary actuation decision (active or inactive). 


\subsection{Input combinations}

With 10 cylinders, there are 10 pressure chambers that may either be active or inactive for a total of $2^{10}=1024$ combinations at every sample. However, due to symmetry it makes no sense to motor and pump simultaneous with different cylinders, such that there is $2^{5}=32$ pumping and motoring combinations. The different combination are shown in Fig. 4 and are illustrated as normalized values (displacement fractions). The relations between the displacement fraction to the flow and torque are given by

$$
Q[k]=\underbrace{\frac{V_{\mathrm{d}} N_{\mathrm{c}}}{2 \pi}}_{k_{\mathrm{q}}} \omega[k] \alpha[k] \quad \tau[k]=\underbrace{\frac{V_{\mathrm{d}} N_{\mathrm{c}}}{2 \pi}}_{k_{\tau}} p_{\mathrm{H}}[k] \alpha[k]
$$

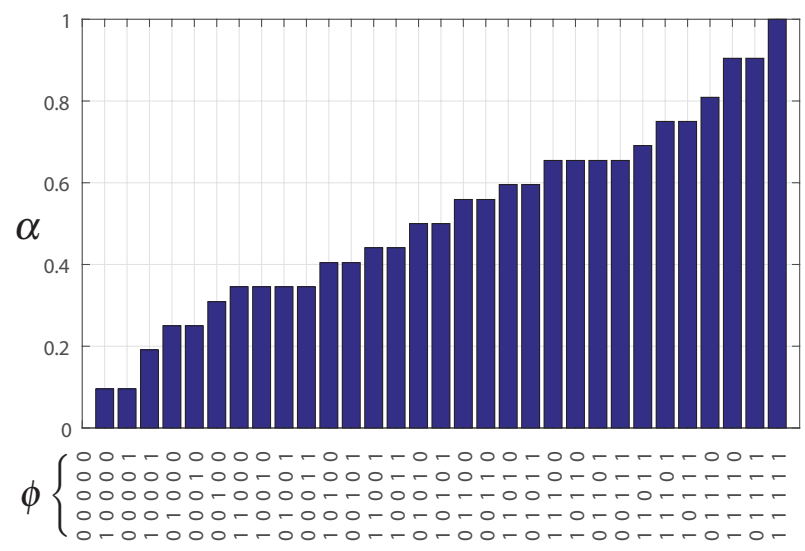

Figure 4: Combinations of discretized displacement fraction.

As expected a displacement fraction of 1 is obtained by activating all five pressure chambers operating in the same mode (motoring or pumping). It is seen that several of the combinations yield the same output, but they may have significantly different energy cost related, since one may require a valve switch while the other may not require a switch. Additionally, it is more energy costly and the flow/pressure spikes are higher when switching at high flow levels.

\subsection{Input mapping}

Having identified an optimal discrete input at a given sample, the next step is to map the input to a pressure chamber actuation sequence for the 10 cylinders. Let the prediction horizon $p=\operatorname{ceil}\left(N_{\mathrm{c}} / 2\right)$ and $\mathrm{j}$ be such that $\theta[j]=\theta(t)$, where $\theta[j] \wedge \theta(t) \in[0 ; 2 \pi]$. Let $\mathrm{k}$ and $\mathrm{n}$ be defined as $m \in\left\{0, \ldots, N_{\mathrm{c}}-1\right\}$ and $n \in$
$\{0, \ldots, p-1\}$ then the chamber actuation sequence is given by

$$
\Phi[m]= \begin{cases}\phi[n] & \text { for } m=(j-n) \bmod N_{\mathrm{c}} \\ 0 & \text { otherwise }\end{cases}
$$

$\Phi$ hence contains information about whether each individual pressure chamber should be active or inactive. If a value in the vector is changed from 0 to 1 , the LPV for the given pressure chamber is closed following an opening of the HPV. Inversely, if a value is changed from 1 to 0 , the HPV is closed following an opening of the LPV. An example of how the chamber actuation vector $\Phi$ is constructed is shown in Fig. 6 .

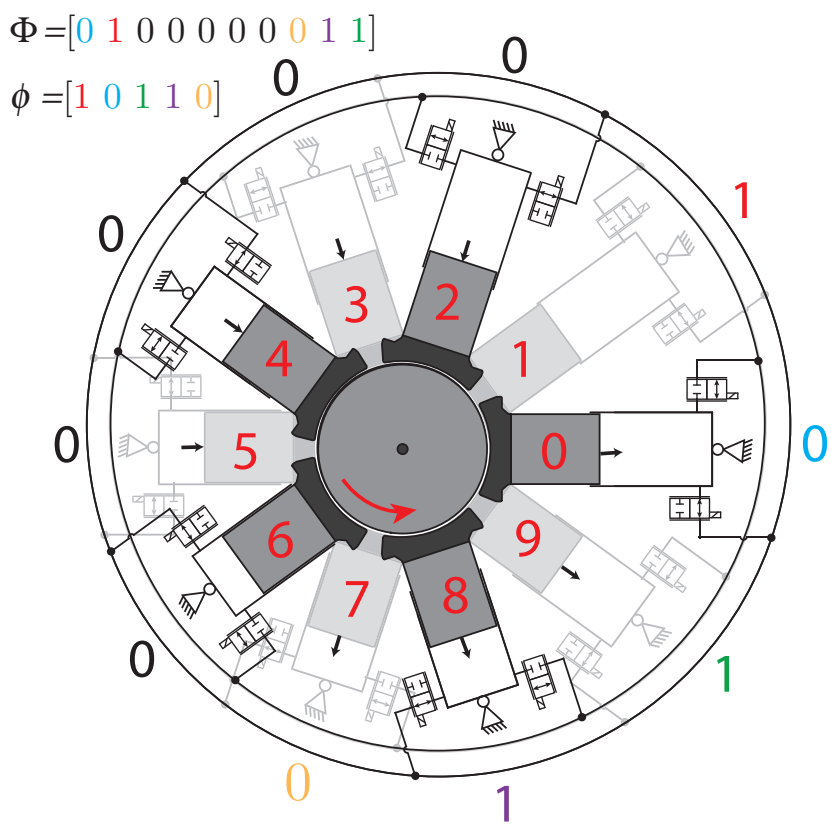

Figure 6: Example of the chamber actuation sequence generation.

In this example cylinder 1 (red) is at Bottom Dead Center (BDC) where a pumping stroke starts. Cylinder $0,9,8$ and 7 are in the pumping stroke part, while the remaining cylinders are in the motoring stroke part. At this sample, $\phi=\left[\begin{array}{lllll}1 & 0 & 1 & 1 & 0\end{array}\right]$ is identified as the optimal input sequence. Since the first entry is 1 , the current cylinder 1 has to be activated, while the previous cylinder 0 has to be inactive given by the second entry. Similarly, the cylinders 9 and 8 have to be active given by the third and fourth entries in $\phi$. Cylinder 7 which has almost completed its pumping stroke should be inactive and the remaining cylinders are inactive as well, since they are in the motoring stroke part. As a result it is seen that $\Phi=[0100000011]$ is the resulting chamber actuation vector, providing information about the state of each pressure chamber. 

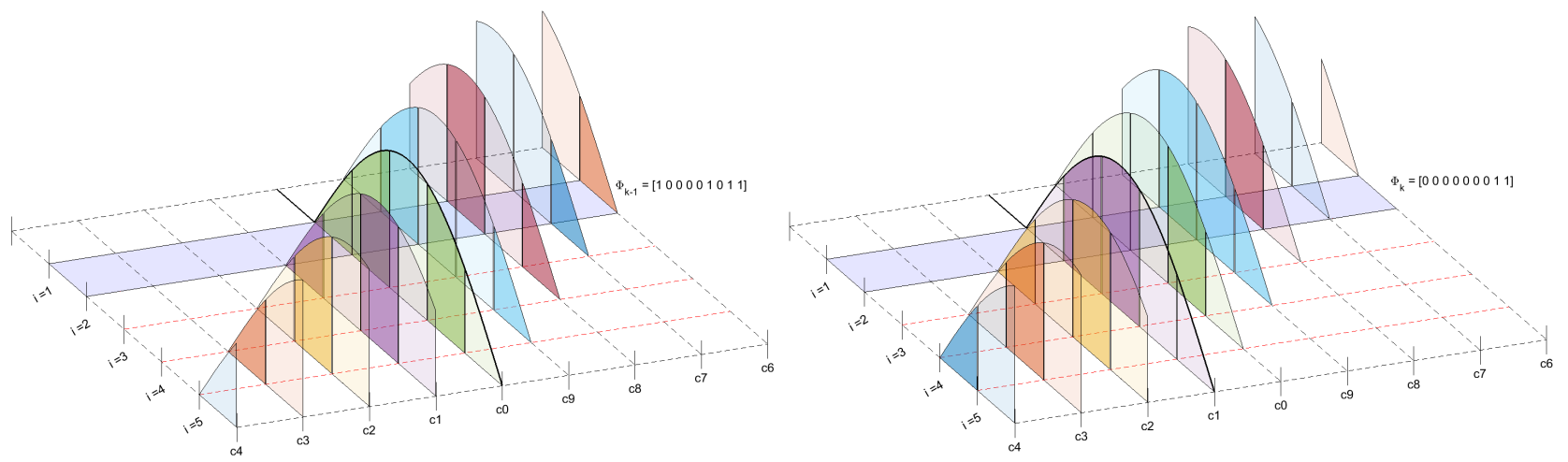

Figure 5: Illustration of the chamber actuation decisions in the prediction horizon for sample $k-1$ and $k$. Those areas shown with a dark color illustrate an active period.

\subsection{MPC flow control}

This paper presents a flow and a pressure control method for a low speed DD pump, where the flow control method is relatively simple since no dynamics is present and the future state estimation is thus omitted. The optimization problem of determining the optimal displacement fraction input is found as a combination of reference tracking accuracy and energy cost given as

$$
\begin{aligned}
\alpha^{*} & =\underset{\alpha^{*} \in \alpha}{\operatorname{argmin}}\left\{J_{1}^{T} J_{1}+W_{1} J_{2}^{T} J_{2}\right\} \\
J_{1} & =\sum_{i=1}^{p}\left(k_{\mathrm{q}} \omega \alpha[i]^{*}-y_{\mathrm{ref}}[i]\right)
\end{aligned}
$$

$\alpha^{*}$ is hence a vector containing the optimal inputs in the prediction horizon. $J_{1}$ is the cost function for the reference tracking and $J_{2}$ for the switching cost, where $W_{1}$ is a scaling parameter between the two cost functions. The tracking error cost function $J_{1}$ is seen to be the sum of errors in the prediction horizon. The energy cost function $J_{2}$ is defined as

$$
\begin{aligned}
J_{2} & =\sum_{i=0}^{N_{\mathrm{c}}-1}\left(\frac{\lambda[i]}{\sum_{j=0}^{N_{\mathrm{c}}-1} \lambda[j]}\left|\Phi[i]_{\mathrm{k}}-\Phi[i]_{\mathrm{k}-1}\right|\right) \\
\lambda[n] & = \begin{cases}1+W_{2} \sin (\theta[n]) & \text { for } m=(j-n) \bmod N_{\mathrm{c}} \\
0 & \text { otherwise }\end{cases}
\end{aligned}
$$

The (k)-index and (k-1)-index of the actuation vector $\Phi$ indicate the current and previous optimized vector. For every value change in the actuation vector, $\Phi$, the correspondent valves for the given pressure chambers are switched and energy cost is added to the function. Since no specific valve design is considered, the base energy cost of a switch is set to 1 by the function $\lambda$, using the same definitions as in (12). The function $\sin (\theta[n])$ takes into account that it costs more to switch at higher flow rates, where also the pressure spikes are larger, and $W_{2}$ is again a scaling parameter. The denominator in $J_{2}$ is introduced to normalize the function, such that the value of $J_{2}$ always has a value between 0 and 1 . An illustration of how the energy cost function is evaluated in the prediction horizon is shown in Fig. 5. It is seen that the optimized input $\alpha^{*}$ corresponds to an actuation sequence for the respective pressure chambers in the prediction horizon. The energy cost function is only evaluated for the current step $i=1$, since only the actuation vector for the current sample determines which valves are switched. The energy cost could be evaluated in the full prediction horizon, but this has been found to yield slightly worse performance. Alternatively, a weighted sum could be used to penalize the early steps in the prediction horizon more than the later ones.

\subsection{Optimization algorithm}

In this paper, the optimal solution to the optimization problem is solved by the differential evolution algorithm (DEA), due to its feature of searching a large space and being able to solve discrete valued problems. Better and faster algorithms for the given problem may very well exist, but this is considered out of scope of this paper. The algorithm has previously been successfully applied to a similar problem for discrete force control of a wave energy converter in Hansen et al. (2017). For more information about the DEA, see e.g. Bech et al. (2016). To show the best possible tracking performance with the proposed control strategy, the energy cost function scaling parameter is initially chosen as $W_{1}=0$, while $W_{2}=10$. The optimized result is shown in Fig. 7. For this relatively easy optimization 

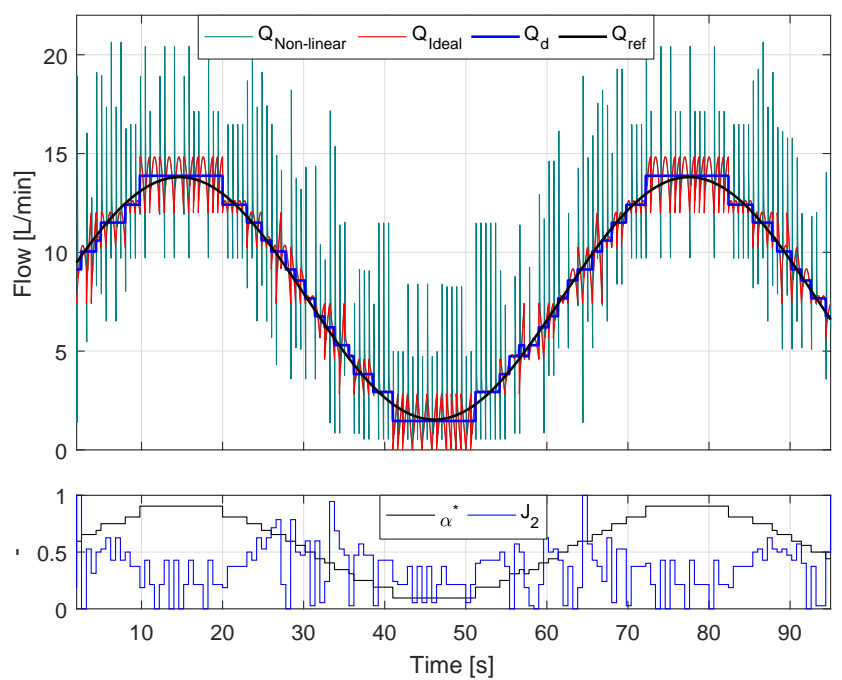

Figure 7: Flow tracking response using the DEA-MPC algorithm without the energy cost function.

problem, the optimal solution could be identified manually by taking the discrete value in Fig. 4 that yields the lowest value when evaluating $\left|k_{\mathrm{q}} \omega \alpha-y_{\text {ref }}\right|$, which gives the same result. $y_{\text {DLTI }}=k_{\mathrm{q}} \omega \alpha_{1}^{*}$ is the chosen discrete value. $y_{\text {ideal }}$ is the ideal flow output considering $Q_{\mathrm{H}}=\dot{V}_{\mathrm{c}}$ and $y_{\mathrm{NLM}}$ is the flow output of the non-linear dynamic model. The results are as expected, where the integrated value of $y_{\text {DLTI }}$ and $y_{\text {ideal }}$ are identically and that of $y_{\mathrm{NLM}}$ is a bit higher due to the flow spikes. The bottom part of Fig. 7 shows the optimal input $u=\alpha_{1}^{*}$ and the switching cost function value of $J_{2}$. It is seen that there are several valve switchings with a high cost that may be unfavorable. When changing the cost scaling parameter to $W_{1}=2 \mathrm{e}-9$, results in the optimized solution shown in Fig. 8. It is evident, that the tracking accuracy has been reduced, especially at low and high flow rates. However, it is seen that the switchings with a high cost have been removed completely. Taking the time integral of the absolute tracking error and energy cost yield the results given in Tab. 2 .

Table 2: Results with and without energy cost.

\begin{tabular}{l|c|c}
\hline Parameter & w/o. $J_{2}$ & w. $J_{2}$ \\
\hline$W_{1}$ & 0 & $2 \mathrm{e}-9$ \\
Integrated absolute error & $1.6 \mathrm{~L}$ & $2.1 \mathrm{~L}$ \\
Integrated energy $\left(J_{2}\right)$ & 96.53 & 57.17 \\
\hline
\end{tabular}

It is seen that the tracking error is $29 \%$ higher when the energy cost is included in the cost function, but the energy cost is $41 \%$ higher without the energy cost included. This is a simple example to illustrate how the optimal solution changes based on the trade off between tracking and energy. A good choice of the scal-

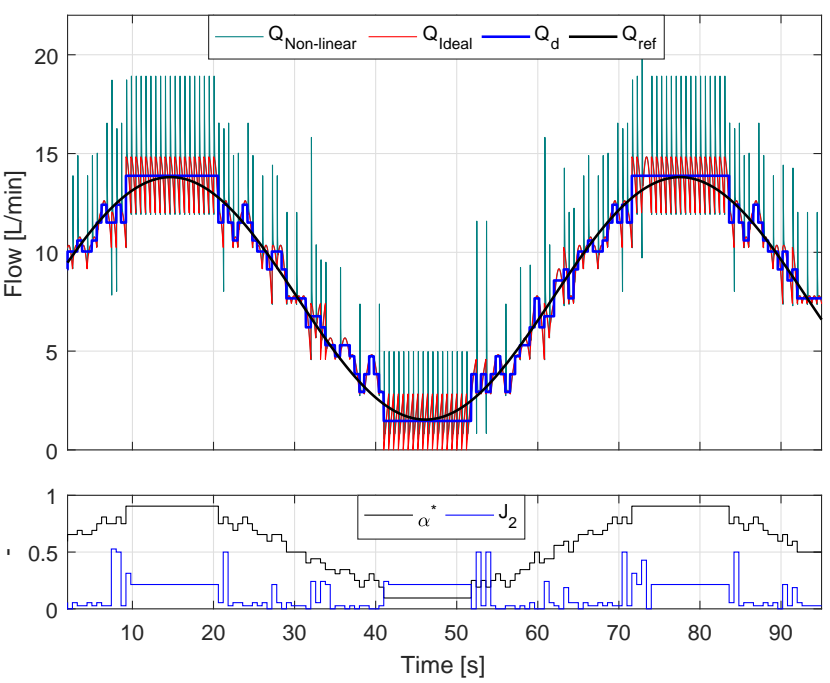

Figure 8: Flow tracking response using the DEA-MPC algorithm with the energy cost function.

ing parameter $W_{1}$ is of course dependent on the control and energy requirements for the specific application.

\subsection{MPC pressure control}

A relatively simple system is constructed in this paper to illustrate the applicability of the control structure, but it is expected that the control method is suitable for more advanced fluid power systems comprising lowspeed digital displacement machines with a relative low number of cylinders. An illustration of the system considered for pressure control is shown in Fig. 9.

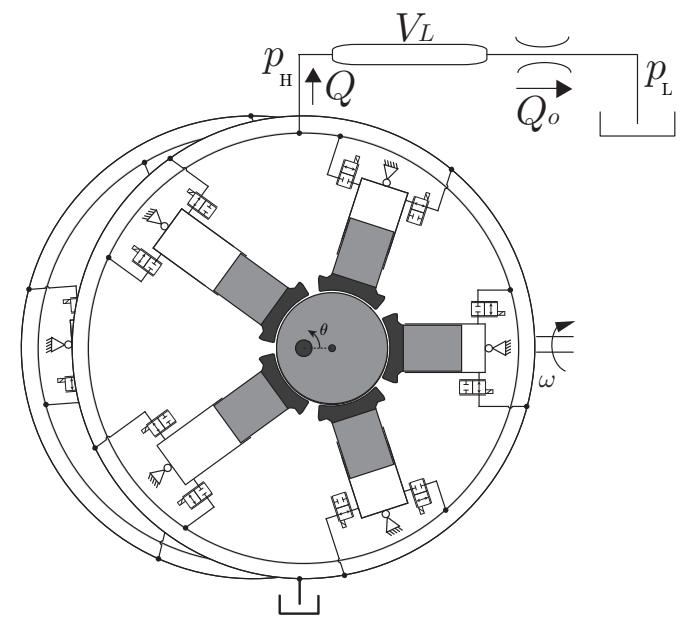

Figure 9: Illustration of the simple load system used for pressure control of the digital displacement machine.

A simple orifice load is considered as a flow restriction, where the objective is to control the pressure in 
the high pressure manifold, $p_{\mathrm{H}}$. For pressure control, a DLTI model of the pressure dynamics is derived based on the shown definition of variables. The dynamic equation describing the pressure is given by

$$
\dot{p}_{\mathrm{H}}(t)=\frac{\beta_{\mathrm{e}}}{V_{\mathrm{L}}}(\underbrace{k_{\mathrm{q}} \omega \alpha(t)}_{Q(t)}-\underbrace{k_{\mathrm{qo}} \sqrt{p_{\mathrm{H}}(t)}}_{Q_{\mathrm{o}}(t)})
$$

where $k_{\mathrm{qo}}=2.2 \mathrm{~L} /(\min \sqrt{\mathrm{bar}}), V_{\mathrm{L}}=0.1 \mathrm{~m}^{3}$ is the volume in the pressure line, $\beta_{\mathrm{e}}$ is the effective bulkmodulus and $u=\alpha$ is the input. Due to the non-linear equation describing the flow through the orifice, a linearization by first order Taylor approximation is made and results in

$$
\underbrace{\delta \dot{p}_{\mathrm{H}}(t)}_{\dot{x}}=\underbrace{-\frac{\beta k_{\mathrm{qo}}}{2 V_{\mathrm{L}} \sqrt{p_{0}}}}_{A_{\mathrm{c}}} \underbrace{\delta p_{\mathrm{H}}(t)}_{x}+\underbrace{\frac{\beta}{V_{\mathrm{L}}} k_{\mathrm{q}} \omega}_{B_{\mathrm{c}}} \underbrace{\delta u(t)}_{u}
$$

A discretization is made by assuming a zero-order-hold input as approximated by the discrete model having the sampling time $T_{\mathrm{s}}=2 \pi /\left(N_{\mathrm{c}} \omega\right)$. The resulting DLTI model has the standard state-space form given by

$$
\begin{aligned}
x_{\mathrm{k}+1} & =A x_{\mathrm{k}}+B u_{\mathrm{k}} \\
y_{\mathrm{k}} & =C x_{\mathrm{k}}
\end{aligned}
$$

It has been identified that integral action added to the control system is necessary to obtain offset-free reference tracking, due to the model inaccuracies caused by linearization and flow discretization. Incorporating integral action within the MPC control structure is done by modifying the state model to a state difference model in accordance with Stephens et al. (2013) and result in

$$
\begin{aligned}
\Delta x_{\mathrm{k}+1} & =A \Delta x_{\mathrm{k}}+B \Delta u_{\mathrm{k}} \\
\Delta y_{\mathrm{k}+1} & =C \Delta x_{\mathrm{k}}
\end{aligned}
$$

where $\Delta x_{\mathrm{k}}=x_{\mathrm{k}}-x_{\mathrm{k}-1}, \Delta u_{\mathrm{k}}=u_{\mathrm{k}}-u_{\mathrm{k}-1}$ and $\Delta y_{\mathrm{k}}=$ $y_{\mathrm{k}}-y_{\mathrm{k}-1}$. Rewriting the output equation yields the desired result given as

$$
\begin{aligned}
\Delta y_{\mathrm{k}+1} & =C \Delta x_{\mathrm{k}} \\
y_{\mathrm{k}+1}-y_{\mathrm{k}} & =C\left(A \Delta x_{\mathrm{k}}+B \Delta u_{\mathrm{k}}\right) \\
y_{\mathrm{k}+1} & =C A \Delta x_{\mathrm{k}}+C B \Delta u_{\mathrm{k}}+y_{\mathrm{k}}
\end{aligned}
$$

It is seen that the output $y_{\mathrm{k}+1}$ is now dependent on the previous output $y_{\mathrm{k}}$ similar to a conventional discrete integrator. Forming the new state formulation yields

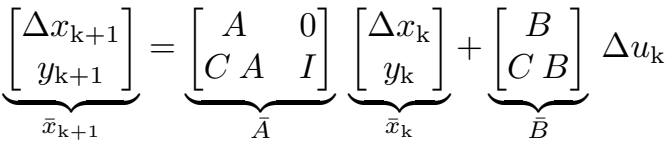

$$
\begin{aligned}
& y(k)=\underbrace{\left[\begin{array}{ll}
0 & I
\end{array}\right]}_{\bar{C}} \bar{x}_{\mathrm{k}}
\end{aligned}
$$

This state model is then used to predict the future state development by use of recursive evaluation. The recursive discrete state estimation method is given by

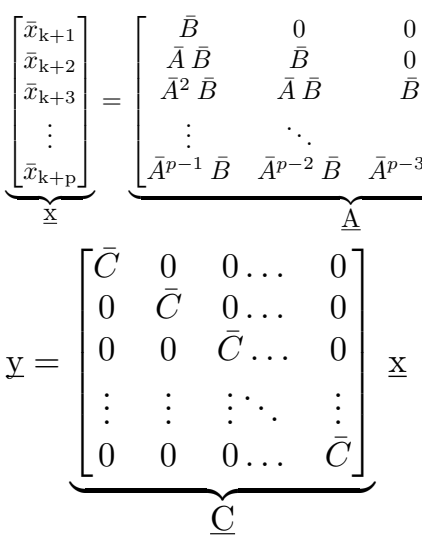

In short notation the discretely predicted pressure may thus be written as

$$
\underline{\mathrm{y}}=\underbrace{\underline{\mathrm{C}} \underline{\mathrm{A}}}_{\Psi} \Delta \underline{\mathrm{u}}+\underbrace{\mathrm{C}}_{\gamma} \underline{\mathrm{B}} \bar{x}_{\mathrm{k}}
$$

The optimization problem is very similar to that for the flow control problem, except for how the predicted output is used to estimate the tracking error cost function. The optimization problem becomes that given as

$$
\begin{aligned}
\underline{\mathrm{u}} & =\underset{\underline{\mathrm{u}} \in \alpha}{\operatorname{argmin}}\left\{J_{1}^{T} J_{1}+W_{1} J_{2}^{T} J_{2}\right\} \\
J_{1} & =\sum_{i=1}^{p}\left(\left(\Psi \Delta_{\underline{\mathrm{u}}_{\mathrm{k}}}[i]+\gamma \bar{x}_{\mathrm{k}}\right)-y_{\mathrm{ref}}[i]\right) \\
J_{2} & =\sum_{i=0}^{N_{\mathrm{c}}-1}\left(\frac{\lambda[i]}{\sum_{j=0}^{N_{\mathrm{c}}-1} \lambda[j]}\left|\Phi[i]_{\mathrm{k}}-\Phi[i]_{\mathrm{k}-1}\right|\right)
\end{aligned}
$$

It should be noticed that the difference input $\Delta u_{\mathrm{k}}=$ $u_{\mathrm{k}}-u_{\mathrm{k}-1}$ has to be evaluated at each function evaluation using the current and previous optimal input vector.

\section{Results}

To investigate the influence of the energy cost function, the control strategy is initially implemented with the energy cost function scaling parameter chosen as $W_{1}=$ 0 , while $W_{2}=10$. The results of the optimized problem is shown in Fig. 10. 

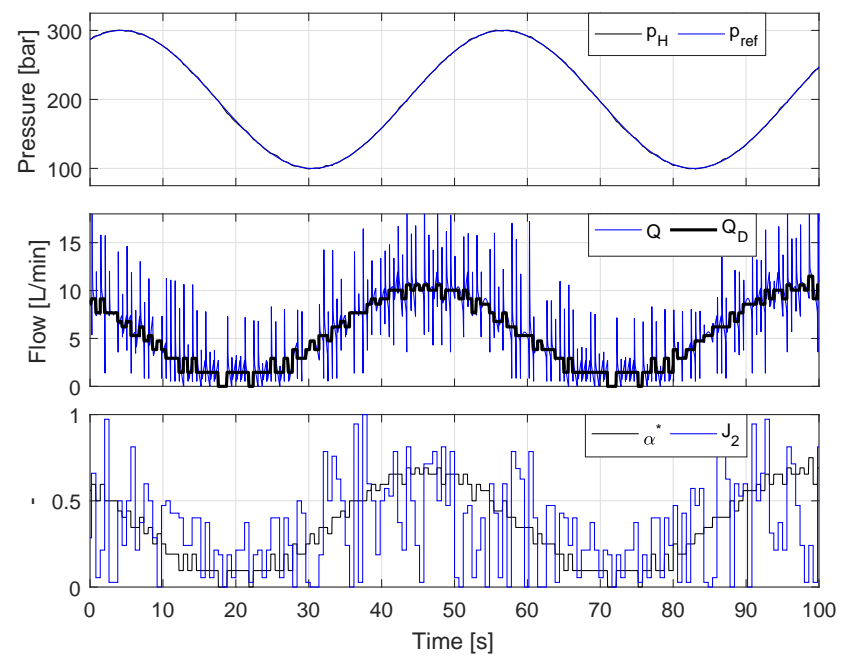

Figure 10: Pressure tracking response using the DEA MPC algorithm without the energy cost function.

It is seen that relative great reference tracking is obtained with a minor amount of ripples around the tracking point due to the non-smooth flow profile. The bottom plot reveals that there is a high number of expensive switchings, where a value of 1 indicates that the maximum of 6 valves are switched simultaneously. When changing the cost scaling parameter to $W_{1}=2 \mathrm{e} 12$, yields the optimized results shown in Fig. 11.
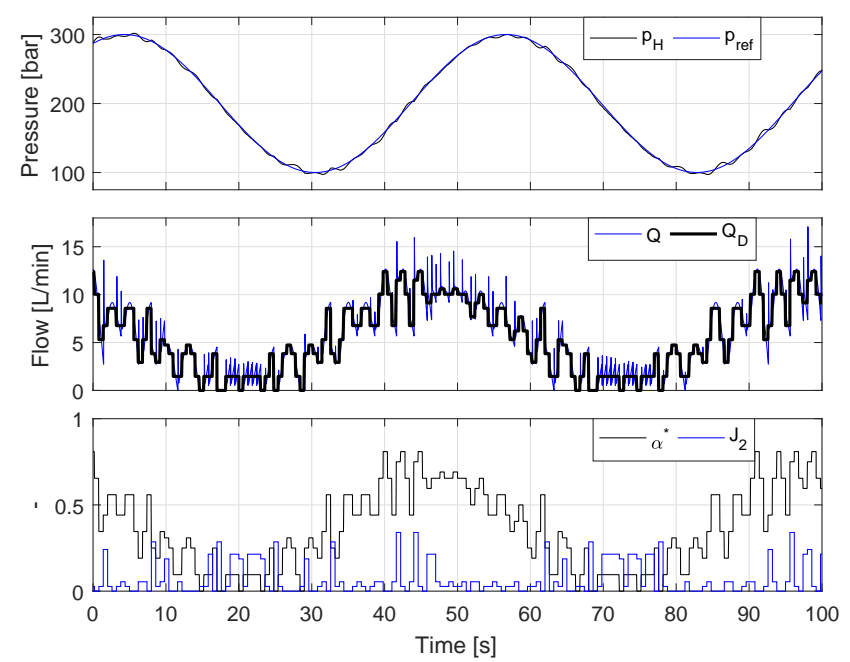

Figure 11: Pressure tracking response using the DEA MPC algorithm with the energy cost function.

As expected the reference tracking error has increased and larger ripples around the set-point is observed. It is also clear that the switching cost has been reduced significantly. Additionally, the large flow spikes due to switching at high flow rates have been removed. Taking the time integral of the absolute tracking error and energy yields the results given in Tab. 3 for the two optimized control problems.

Table 3: Results with and without energy cost.

\begin{tabular}{l|c|c}
\hline Parameter & w/o. $J_{2}$ & w. $J_{2}$ \\
\hline$W_{1}$ & 0 & $2 \mathrm{e} 12$ \\
Integrated absolute error & $2.27 \mathrm{e} 7$ & $2.95 \mathrm{e} 7$ \\
Integrated energy & 30.3 & 6.1 \\
\hline
\end{tabular}

It is seen that the tracking error is $30 \%$ higher when the energy cost is included in the cost function, but the energy cost is $397 \%$ higher without the energy cost included. For this particular example it is clear that it might be beneficial to penalize the energy cost unless there is strict requirement to set-point tracking. Based on the specific application it is considered easy to adjust the cost function scaling parameter $W_{1}$ based on the desired trade-off between tracking performance and energy cost. In all of the presented results for both flow and pressure control, the problem has been solved in much less time than the control sampling time, which enables the algorithm to be implemented online. However, MPC control requires the reference trajectory to be pre-specified or at least estimated within the prediction horizon which may cause problems based on the specific application. Also, there is a clear restriction with regard to the maximum speed of the digital displacement machine, both due to the problem solving time and valve switching time. Additionally, an increased sample rate may be beneficial for very low speed machines, but this comes with the cost of an increased number of input decisions, which again increases the problem-solving time.

\section{Conclusion}

A Model Predictive Control (MPC) method for a sequential partial stroke operated low speed Digital Displacement Machine (DDM) with a relatively low number of cylinders is demonstrated in this work. The method improves the set-point tracking capability compared to full-stroke and conventional partial stroke operated machines, while allowing the designer to specify the importance of energy consumption and reduced flow/pressure/torque pulsations. To solve the discrete valued optimization problem, the Differential Evolution Algorithm (DEA) is used. For a 10-rpm machine with 10 cylinders the algorithm is online capable, since the solving time is much less than the control sampling time. Simulation results for both flow and pressure control show great tracking performance if energy 
consumption is not considered, while a minor decrease in tracking performance leads to a large decrease in energy consumption in the case of pressure control. The presented control strategy is however not suitable for fast rotating digital displacement machines due to both solving time and additional energy losses due to excessive switchings compared to a full-stroke control method.

\section{Acknowledgments}

This research was funded by the Danish Council for Strategic Research through the HyDrive project at Aalborg University, at the Department of Energy Technology (case no. 1305-00038B).

\section{References}

Armstrong, B. S. R. and Yuan, Q. Multilevel control of hydraulic gerotor motor and pumps. Proceedings of the 2006 American Control Conference Minneapolis, Minnesota, June 2006. doi:10.1109/ACC.2006.1657450.

Bech, M. M., Noergaard, C., Roemer, D. B., and Kukkonen, S. A global multi-objective optimization tool for design of mechatronic components using generalized differential evolution. Proceedings of the 42nd Annual Conference of IEEE Industrial Electronics Society, 2016. doi:10.1109/IECON.2016.7793419.

Hansen, A. H., Asmussen, M. F., and Bech, M. M. Energy optimal tracking control with discrete fluid power systems using model predictive control. The Ninth Workshop on Digital Fluid Power, September 7-8, Aalborg, Denmark, 2017.

Heikkila, M. and Linjama, M. Displacement control of a mobile crane using digital hydraulic power management system. Mechatronics 23(4), pp. 452 - 461, 2013. doi:10.1016/j.mechatronics.2013.03.009.

Johansen, P. Tribodynamic Modeling of Digital Fluid Power Motors. Ph.D. thesis, Department of Energy Technology, Aalborg University, 2014.

Johansen, P., Roemer, D. B., Pedersen, H. C., and Andersen, T. O. Delta-sigma modulated displacement of a digital fluid power pump. Proceedings of the rth Workshop on Digital Fluid Power, Linz, Austria, 2015. The Seventh Workshop on Digital Fluid Power, Linz, Austria.
Johansen, P., Roemer, D. B., Pedersen, H. C., and Andersen, T. O. Discrete linear time invariant analysis of digital fluid power pump flow control. Journal of Dynamic Systems, Measurement and Control, Transactions of the ASME, Vol. 139, Nr. 10, 101007, 2017. doi:10.1115/1.4036554.

Linjama, M. Digital fluid power state of the art. The Twelfth Scandinavian International Conference on Fluid Power, May 18-20, Tampere, Finland, 2011.

M. Ehsan, W. R. and Salter, S. Modeling of digital-displacement pump-motors and their application as hydraulic drives for nonuniform loads. ASME, Journal of dynamic system measurement and control, Vol. 122, pp. 210-215, March 2000. doi:10.1115/1.482444.

Merrill, K., Holland, M., and Lumkes, J. Analysis of digital pump/motor operating strategies. Proceedings of the 52nd National Conference on Fluid Power, 2011.

Noergaard, C. Design, Optimization and Testing of Valves for Digital Displacement Machines. Ph.D. thesis, Aalborg University, 2017. doi:10.5278/vbn.phd.eng.00013.

Payne, G. S., Stein, U. P. P., Ehsan, M., Caldwell, N. J., and Rampen, W. H. S. Potential of digital displacement hydraulics for wave energy conversion. In Proc. of the 6th European Wave and TIdal Energy Conference, Glasgow UK., 2005.

Pedersen, N. H., Johansen, P., and Andersen, T. O. Event-driven control of a speed varying digital displacement machine. Proceedings of ASME/BATH FPMC Symposium on Fluid Power and Motion Control, Sarasota, Florida, USA, 2017a. doi:10.1115/FPMC2017-4260.

Pedersen, N. H., Johansen, P., and Andersen, T. O. Lqr-feedback control development for wind turbines featuring a digital fluid power transmission system. 9th FPNI PhD symposium on fluid power, Florianpolis, Brazil, 2017b. doi:10.1115/FPNI2016-1537.

Pedersen, N. H., Johansen, P., and Andersen, T. O. Challenges with respect to control of digital displacement hydraulic units. Modeling, Identification and Control, Vol 39, 2018a. doi:10.4173/mic.2018.2.4.

Pedersen, N. H., Johansen, P., and Andersen, T. O. Optimal control of a wind turbine with digital fluid power transmission. Nonlinear Dynamics, 2018b. doi:10.1007/s11071-017-3896-0. 
Rampen, W. The development of digital displacement technology. In Proceedings of Bath/ASME FPMC Symposium, 2010.

Roemer, D. B. Design and Optimization of Fast Switching Valves for Large Scale Digital Hydraulic Motors. Ph.D. thesis, 2014. Department of Energy Technology, Aalborg University.

Sniegucki, M., Gottfried, M., and Klingauf, U. Optimal control of digital hydraulic drives using mixedinteger quadratic programming. 9th IFAC Symposium on Nonlinear Control System, Toulouse, 2013. doi:10.3182/20130904-3-FR-2041.00013.

Song, X. Modeling an active vehicle suspension system with application of digital displacement pump motor. Proceedings of the ASME 2008 International Design Engineering Techical Conference/Computers and Information in Enegineering Conference, Brooklyn, New York, 2008. doi:10.1115/DETC2008-49035.

Stephens, M. A., Manzie, C., and Good, M. C. Model predictive control for reference tracking on an industrial machine tool servo drive. IEEE Transactions on Industrial Informatics, Vol. 9, 2013. doi:10.1109/TII.2012.2223222.

Wilfong, G., Batdorff, M., and Lumkes, J. Design and dynamic analysis of high speed on/off poppet valves for digital pump/motors. In Proceedings of the 6th FPNI-PhD Symposium, 2010.

Wilfong, G., Holland, M., and Lumkes, J. Design and analysis of pilot operated high speed on/off valves for digital pump/motors. In Proceedings of the 52nd National Conference on Fluid Power, 2011. 